\title{
COVERING AND FUNCTION THEORETIC PROPERTIES OF UNIFORM SPACES
}

\author{
BY MICHAEL D. RICE
}

Communicated by W. W. Comfort, May 21, 1973

The purpose of this note is to announce the major ideas and results developed in $[\mathbf{R}]_{1}$. The proofs of these results will appear in a series of three papers $[\mathbf{R}]_{2},[\mathbf{R}]_{3}$, and $[\mathbf{R R}]$, the latter including categorical topics that will be omitted here. The subject matter is the covering and function theoretic properties of uniform spaces, a subject initiated by John Isbell in the 1950's. (See [GI] and [I].) Our work represents a continuation and extension of the current work of Anthony Hager $\left([\mathbf{H}]_{1},[\mathbf{H}]_{2}\right)$ and $\mathbf{Z}$. Frolik; and overlaps somewhat with recent work of Z. Frolik $\left([\mathbf{F r}]_{1},[\mathrm{Fr}]_{2}\right)$. The author wishes to emphasize that his work substantiates the existence of a theory of uniform structures which is not primarily interested in topological applications. Therefore, the viewpoint adopted here is one of intrinsic interest per se in uniform properties.

A uniform space is denoted by $u X$, where $u$ is a family of covers on the set $X$ constituting a uniformity. $u X$ is fine if $u$ is the largest uniformity on $X$ with the same uniform topology. A subfine space is a subspace of a fine space. $u X$ is locally fine if each cover of the form $\left\{A_{\alpha} \cap C_{\beta}^{\alpha}\right\} \in u$, where $\left\{A_{\alpha}\right\} \in u$, and $\left\{C_{\beta}^{\alpha}\right\} \in u$ for each $\alpha . u X$ is $M$-fine (sub-M-fine) if each uniformly continuous function (map) to a metric (complete metric) space remains a map relative to the fine uniformity on $M$ (the uniformity with the basis of open covers of $M) . u X$ is hereditarily $M$-fine if each subspace is $M$-fine.

The basic source on locally fine and subfine spaces is [I], while the development of separable $M$-fine and separable hereditarily $M$-fine spaces (those with a basis of countable covers) originates in $[\mathbf{H}]_{1}$ and $[\mathbf{H}]_{2}$.

One easily sees that each fine space is $M$-fine and that each $M$-fine space is sub- $M$-fine. Example $\mathrm{C}$ of [GI] is a hereditarily $M$-fine space which is not locally fine. [I] shows that each locally fine space is sub- $M$-fine and that each subfine space is locally fine; the converse of the latter is an unsolved problem. From [I] we also know that each separable sub- $M$-fine space is subfine.

AMS (MOS) subject classifications (1970). Primary 54E15; Secondary 54E35. 
The properties we have defined are closed under completion, the formation of sums and quotients, and with the exception of $M$-fine are hereditary properties.

Each of the above properties defines a coreflective subcategory of uniform spaces (given $X$, there exists $X_{\mathscr{C}} \in \mathscr{C}$ and a map $X_{\mathscr{C}} \rightarrow X$ with this property: For each map $Y \rightarrow^{f} X$, with $Y \in \mathscr{C}$, there exists a unique map $Y \rightarrow{ }^{g} X$ such that $\left.\xi g=f\right)$. Actually coreflections have a simpler description here, from which there exists a smallest $M$-fine (resp. sub- $M$-fine, hereditarily $M$-fine, locally fine) uniformity $m u$ (resp. $m_{1} u, m_{*} u, \lambda u$ ) containing $u$. The following is evidently a method for generating the uniformities $m u$ and $m_{1} u$.

Let $\left\{X \rightarrow^{f} \alpha M: u X \rightarrow^{f} \rho M\right.$ is a map, $\rho M$ metric (complete metric) generate $\omega u\left(\omega_{1} u\right)$, where $\alpha$ is the fine uniformity on $M$. Inductively, if $\beta$ is a limit ordinal, let $\omega^{(\beta)} u=\bigcup\left\{\omega^{(\gamma)} u: \gamma<\beta\right\}$; otherwise let $\omega^{(\beta)} u=$ $\omega\left(\omega^{(\beta-1)} u\right)$. Then $m u=\bigcup \omega^{(\beta)} u$ and $m_{1} u=\bigcup \omega_{1}^{(\beta)} u$. Elaborating a technique from [V], we can show the extra steps unnecessary and from 1.1 we can then justify the term "sub- $M$-fine".

THEOREM 1.1. $\quad m u=\omega u ; m_{1} u=\omega_{1} u$.

THEOREM 1.2. The sub-M-fine spaces are precisely the subspaces of M-fine spaces.

COROLlARY 1.3. Each locally fine space is a subspace of an M-fine space.

Actually 1.1 may be considerably improved by a covering characterization of $m u$ and $m_{1} u$. We first examine the separable case. If we restrict ourselves to separable (complete separable) metric spaces in the definition of $M$-fine (sub- $M$-fine), and denote the analogous modifications $m^{s}, m_{1}^{s}$, $m_{*}^{s}$, we have this characterization, where $\vee$ denotes the least upper bound operation and $e u$ is the uniformity with the basis of countable covers from $u$.

THEOREM 2.1. (a) $m^{s} u=u \vee m e u$. (b) $m_{1}^{s} u=u \vee m_{1} e u=u \vee \lambda e u$. (c) $m_{*}^{s} u=$ $u \vee m_{*} e u$.

THEOREM 3.1. $\quad m u\left(m_{*} u\right)$ has the basis of covers of the form $\left\{A_{n} \cap C_{\alpha}^{n}\right\}$, where $\left\{A_{n}\right\} \in \operatorname{meu}\left(m_{*} e u\right)$ and $\left\{C_{\alpha}^{n}\right\} \in u$.

Theorems 2.1 and 3.1 have been independently achieved by Z. Frolík. $\left(\right.$ See $[\mathbf{F r}]_{1},[\mathbf{F r}]_{2}$.) In $[\mathbf{H}]_{1}$ an explicit description of meu and $m_{*} e u$ is given: $\operatorname{meuX}\left(m_{*} e u X\right)$ has a basis of covers of the form $\left\{\operatorname{coz}\left(f_{n}\right): f_{n} \in C(u X)\right\}$ $\left(\left\{A_{n}: A_{n} \in \sigma(\operatorname{coz} C(u X))\right\}\right)$, where $\sigma(\operatorname{coz} C(u X))$ is the $\sigma$-algebra generated by the family $\{\operatorname{coz}(g): g \in C(u X)\}$ and $\operatorname{coz}(g)=\{x: g(x) \neq 0\}$. 
THEOREM 3.2. If $\lambda e \rho M=e \alpha M$ for each complete metric space $\rho M$, then $m_{1} u$ has the basis of covers of the form $\left\{A_{n} \cap C_{\alpha}^{n}\right\}$, where $\left\{A_{n}\right\} \in m_{1} e u=$ $\lambda e u$, and $\left\{C_{\alpha}^{n}\right\} \in u$. (Here $\alpha$ denotes the fine uniformity on $M$.)

Call the spaces which are characterized by 3.2 locally sub-M-fine and denote the associated operation $m_{0}$. These are precisely the spaces for which $\left\{A_{n} \cap C_{\alpha}^{n}\right\} \in u$, when $\left\{A_{n}\right\} \in e u$ and $\left\{C_{\alpha}^{n}\right\} \in u$.

Proposition 3.3. (a) $m e=e m$. (b) $m_{*} e=e m_{*}$. (c) $m_{0} e=e m_{0}$.

(a) and (b) have been independently achieved in $[\mathbf{F r}]_{1}$.

Once again, I do not know if 3.3 holds for $m_{1}$; of course if 3.2 holds this will be the case and $m_{0}$ will be $m_{1}$. We can prove

Proposition 3.4. Each sub-M-fine space is locally sub-M-fine.

THEOREM 3.5. $u X$ is $M$-fine (hereditarily $M$-fine) if and only if $u X$ is locally sub-M-fine and $C(u X)$ is closed under inversion (a regular ring).

$C(u X)$ is closed under inversion when $f \in C(u X), f \neq 0$, implies that $1 / f \in C(u X)$. The separable case of 3.5 may be found in $[\mathbf{H}]_{1}$.

THEOREM 4.1. If $u X$ is locally fine, $m u X$ and $m_{*} u X$ are locally fine; hence $m_{*} \rho M$ is locally fine for each metric space $\rho M$.

I have been unable to determine if $\lambda$ preserves the $M$-fine property. If this is the case, then 2.3 shows that each locally fine space is a subspace of a locally fine $M$-fine space; hence the unsolved question reduces to whether each locally fine $M$-fine space is subfine.

We turn now to the closed subspaces of products of metric spaces, which for convenience will be called metric complete. The fundamental connection with the notion of $M$-fine is given by 5.1 .

THEOREM 5.1. The following are equivalent.

(a) muX is complete.

(b) $u X$ is metric complete.

(c) Each u-Cauchy filter with the countable intersection property converges.

THEOREM 5.2. The following are equivalent.

(a) $u X$ is metric complete (with the property that $X$ has no closed discrete subspace of Ulam measurable power).

(b) euX and cuX are each isomorphic to a closed subspace of a product of separable metric spaces.

THEOREM 5.3. A precompact space is metric complete if and only if it is isomorphic to a closed subspace of powers of $(0,1)$. 
Let $d$ denote the functor which reflects uniform spaces into metric complete spaces. We have this description, where $\pi u X$ denotes the completion of $u X$.

THEOREM 5.4. $d u X$ is the $G_{\delta}$ closure of $X$ in $\pi u X$.

In [RR] $d u X$ is described in terms of a natural inverse limit associated with $u X$. This description has been obtained by Morita $[\mathbf{M}]$, and a similar one in topology by Zenor [Z]. In [H] Husek has characterized the precompact metric complete spaces in a different manner.

THEOREM 5.5. The functors $m$ and $d$ commute: $m d=d m$.

Finally, we conclude with a short discussion of covering properties.

THEOREM 6.1. If $u X$ is complete (with the cardinal restriction of 6.2(a)) and has a basis of point finite (star finite) uniform covers, then euX $(\mathrm{cuX})$ is complete.

This result (apparently) generalizes the analogous result found in [GI] for spaces with a $\sigma$-disjoint basis, since each $\sigma$-point finite uniform cover has a point finite uniform refinement. ${ }^{1}$ However, no example of a uniform space without a $\sigma$-disjoint basis is known. We do have the following results.

THEOREM 6.2. A locally sub-M-fine space has a point finite basis if and only if it has a $\sigma$-disjoint basis.

Proposition 6.3. Each sub-M-fine space has a $\sigma$-disjoint basis; each hereditarily $M$-fine space has a basis of disjoint uniform covers.

It is unknown whether the restriction of a point finite basis in 6.1 is needed to insure $e u X$ complete. If each locally sub-M-fine space has a point finite basis, the question can be answered negatively (since $u X$ is complete if and only if $m_{0} u X$ is complete, 3.3(c) and 6.1 insure $e m_{0} u X=$ $m_{0} e u X$ complete; hence $e u X$ is complete). Finally, example B of [GI] is a complete space $u X$ for which $c u X$ is not complete.

\section{BIBLIOGRAPHY}

$[\mathrm{Fr}]_{1} \mathrm{Z}$. Frolík, Interplay of measurable and uniform spaces, Proc. Second Internat. Topology Conference in Yugoslavia, Budova, 1972.

$[\mathrm{Fr}]_{2}-$, Measurable uniform spaces, Proc. Second Internat. Topology Conference in Pittsburgh, 1972.

\footnotetext{
${ }^{1}$ This appears in [S] with what the author feels is an incomplete proof. In [RR] another proof is given.
} 
[GI] S. Ginsburg and J. Isbell, Some operators on uniform spaces, Trans. Amer. Math. Soc. 93 (1959), 145-168. MR 22 \#2977.

$[\mathbf{H}]_{1}$ A. Hager, Some nearly fine uniform spaces, Proc. London Math. Soc. (to appear).

$[\mathrm{H}]_{2} \longrightarrow$, Measurable uniform spaces, Fund. Math. 77 (1972), 51-73.

[H] M. Hušek, The class of k-compact spaces is simple, Math Z. 110 (1969), 123-126. MR 39 \#6260.

[I] J. Isbell, Uniform spaces, Math. Surveys, no. 12, Amer. Math. Soc., Providence, R.I., 1964. MR 30 \#561.

[M] K. Morita, Topological completions and M-spaces, Sci. Rep. Tokyo Kyoiku Daigaku Sect. A 10 (1970), 271-288. MR 42 \#6785.

$[\mathbf{R}]_{1}$ Michael D. Rice, Covering and function theoretic properties of uniform spaces, Thesis, Wesleyan University, Middletown, Conn., 1973.

$[\mathbf{R}]_{2} \longrightarrow$, M-fine uniform spaces (in preparation).

$[\mathbf{R}]_{3} \longrightarrow$, Subcategories of uniform spaces, Trans. Amer. Math. Soc. (to appear).

$[\mathbf{R}]_{4} \longrightarrow$, Complete uniform spaces, Proc. Second Internat. Topology Conference in Pittsburgh, 1972.

[RR] Michael D. Rice and George D. Reynolds, Covering properties of uniform spaces, Oxford Quart. J. (to appear).

[S] J. C. Smith, Refinements of Lebesgue covers, Fund. Math. 20 (1971).

[V] G. Vidossich, Two remarks on A. Gleason's factorization theorem, Bull. Amer. Math. Soc. 76 (1970), 370-371. MR 41 \#1021.

[Z] P. Zenor, Extending completely regular spaces with inverse limits, Glasnik Mat. Ser. III 5 (25) (1970), 157-162. MR 43 \#1128.

Department of Mathematics, Ohio University, Athens, Ohio 45701 